Check for updates

Cite this: RSC Adv., 2017, 7, 52621

Received 3rd September 2017

Accepted 6th November 2017

DOI: 10.1039/c7ra09798k

rsc.li/rsc-advances

\section{A near-infrared phosphorescent iridium(III) complex for imaging of cysteine and homocysteine in living cells and in vivo $\uparrow$}

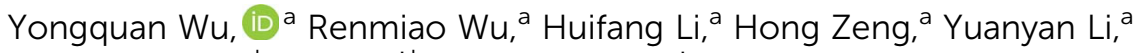 \\ Qiuhong Wang, ${ }^{b}$ Mei Shi*b and Xiaolin Fan*a
}

Based on the cyclization reaction of an aldehyde with an aminoalkyl thiol, a novel NIR-emitting iridium(III) complex (NIR-Ir) showed off-on phosphorescent response toward Cys and Hcy, and was developed to detect Cys/Hcy levels in vitro and in vivo.
Biological thiols (RSH), such as cysteine (Cys) and homocysteine (Hcy), play very crucial roles in physiological processes to maintain the biological redox homeostasis because of their equilibrium between RSH and RSSR. ${ }^{1}$ However, many diseases are related to unusual contents of Cys or Hcy in the human body. Cys deficiency may lead to various symptoms including skin lesions and liver damage. ${ }^{2,3}$ Furthermore, homocysteine is a risk factor for Alzheimer's and cardiovascular diseases., ${ }^{4,5}$ Hence, great attention has been paid to the detection of Hcy and Cys.

Fluorescent detection has emerged as an attractive tool for the detection of various biological molecules, such as thiols, ${ }^{6,7}$ because of its high sensitivity, selectivity, simplicity and its potential application to not only in vitro assays but also in vivo imaging studies. For example, Strongin's group has reported a xanthene dye containing aldehyde that afforded thiazolidines or thiazinanes by reaction with Cys or Hcy, resulting in a decrease in fluorescence emission. ${ }^{8}$

Compared to organic molecules, iridium(III) complexes as chemosensors provide several advantages, such as high photoluminescent efficiencies, relatively long lifetimes and significant Stokes shifts. ${ }^{9,10}$ Hence, many good performances were obtained in the assay of cations, anions, biological molecules and as biological labeling regents. ${ }^{11-23}$ Recently, iridium(III) complex as probe of thiol detection, Che and co-workers developed a FRET-based luminescent iridium(III) complexes for sensing both cysteine and homocysteine. ${ }^{24}$ Ma's group developed a long lifetime iridium(III) complex chemosensor for the selective detection of cysteine. ${ }^{25} \mathrm{Li}$, Zhao, Huang, Yi and

${ }^{a}$ School of Chemistry and Chemical Engineering, Key Laboratory of Organo-pharmaceutical Chemistry of Jiangxi Province, Gannan Normal University, Ganzhou, 341000, P. R. China. E-mail: fanxl2013@gnnu.cn

${ }^{b}$ Department of Chemistry, Fudan University, Shanghai 200433, P. R. China. E-mail: shimei@fudan.edu.cn

$\dagger$ Electronic supplementary information (ESI) available: Complex synthesis and characterization and supplementary data. See DOI: 10.1039/c7ra09798k co-workers reported a series of iridium(III) complexes for detection and imaging of homocysteine or both homocysteine and cysteine..$^{26-34}$ Additionally, Chao's group reported an azobis(2,20-bipyridine)-bridged dinuclear iridium(III) complex for thiol imaging. ${ }^{35}$ Other iridium(III) complexes have been designed and synthesized for thiol detection. ${ }^{36-39}$

In order to deeply understand the roles of Cys/Hcy played in biological system, it is crucial to monitor them not only in vitro assays, but also in living cells and living animal. However, most of iridium(III) complexes emit relatively short wavelengths $\left(\lambda_{\mathrm{em} \max }<650 \mathrm{~nm}\right)$, limiting their use in deep-tissue and living animal imaging because of the shallow penetration depth. ${ }^{11}$ Compared to visible light, the advantage of NIR light was employed in biological imaging due to minimum photodamage to biological samples, deep tissue penetration, and minimum interference from background autofluorescence in the living systems. ${ }^{40-42}$ Currently, iridium(III) complexes are underdeveloped in the NIR region and few have been used in bioimaging. ${ }^{43,44}$ Therefore, it remains a challenging task to develop iridium(III) complex probes having NIR emission for the detection and in vivo imaging of Cys and Hcy.

Herein, a novel NIR-emitting iridium(III) complex [(bbt-pqCHO $)_{2}$ Irphen $]^{+} \mathrm{PF}_{6}{ }^{-}$(NIR-Ir) possessing a Cys/Hcy recognition unit, an aldehyde moiety in the main $\mathrm{C}^{\wedge} \mathrm{N}$ ligands bbt-pq as a Cys/Hcy chemosensor (Scheme 1). Hcy/Cys can be sensitively

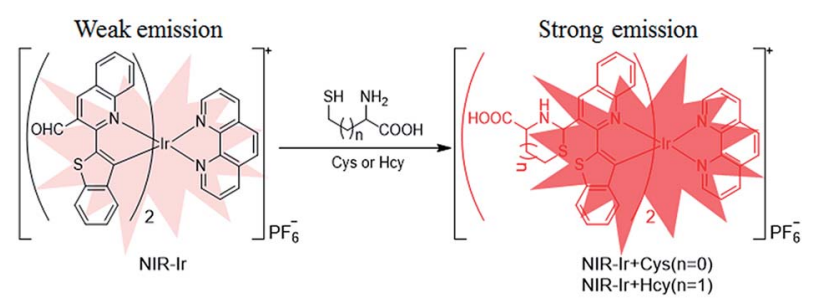

Scheme 1 Chemical structure of NIR-Ir and sensing mechanism of NIR-Ir for Cys and Hcy. 
and selectively detected using the aldehyde bearing biscyclometalated Ir(III) complex as turn-on probe. Therefore, photoluminescence intensity of NIR-Ir increased in the presence of Hcy/Cys based on the special cyclization reaction of aldehyde with aminoalkyl thiol groups. Moreover, NIR-Ir could be applied for the detection and imaging of Cys/Hcy in living animal due to its NIR emission, indicating its significant potential for biological applications.

The synthesis procedures for ligand L1 and NIR-Ir are outlined in Scheme S1 (ESI $\dagger$ ). The ligand L1 was easily obtained by the Suzuki coupling reaction of 2-chloroquinoline-3carbaldehyde with benzo[ $b]$ thiophen-2-boronic acid. The experimental details and characterization data for NIR-Ir are listed in the ESI. $\dagger$

The complex NIR-Ir displays intense absorption band below $400 \mathrm{~nm}$, which should be attributed to the spin-allowed $\mathrm{C}^{\wedge} \mathrm{N}$ ligand-centered LC $\left(\pi-\pi^{*}\right)$ transitions (Fig. S1, ESI $\dagger$ ). The absorption band in the range of $450-600 \mathrm{~nm}$ is assigned to the metal-to- $\mathrm{C}^{\wedge} \mathrm{N}$ ligand charge transfer (MLCT) and $\mathrm{C}^{\wedge} \mathrm{N}$ ligand to $\mathrm{N}^{\wedge} \mathrm{N}$ ligand charge transfer (LLCT) transitions. ${ }^{28}$ The phosphorescent emission range of NIR-Ir lay between 610 and $760 \mathrm{~nm}$ with maximal wavelength at $680 \mathrm{~nm}$, which belongs to the NIR region (650-900 $\mathrm{nm}$ ). The photophysical data of NIR-Ir were summarized in Table S1 (ESI $\dagger$ ). It should be noted that the absorption and emission bands for NIR-Ir were extend to $590 \mathrm{~nm}$ and $760 \mathrm{~nm}$, respectively, which afford an advantage for in vivo imaging.

The detection of Cys and Hcy by NIR-Ir was examined using a spectrophotometric method. The variation of NIR-Ir absorption spectra in DMOS-HEPES (pH 7.4, $4: 1 \mathrm{v} / \mathrm{v}$ ) upon addition of Cys or Hcy was displayed in Fig. 1. As shown in Fig. 1a, with the addition of Cys or Hcy, the absorbance at 338 decreased gradually and the increase at $495 \mathrm{~nm}$. At the same time, the emission intensity at $684 \mathrm{~nm}$ of NIR-Ir increased significantly with Cys/ Hcy concentration (Fig. 1b). According to previous report, the aldehyde group in NIR-Ir was a strong electron-with-drawing
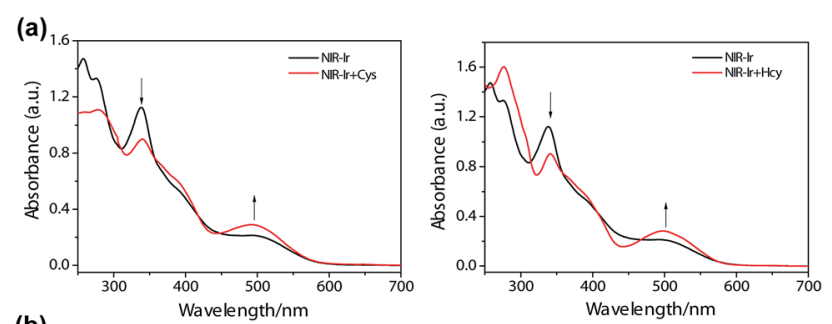

(b)
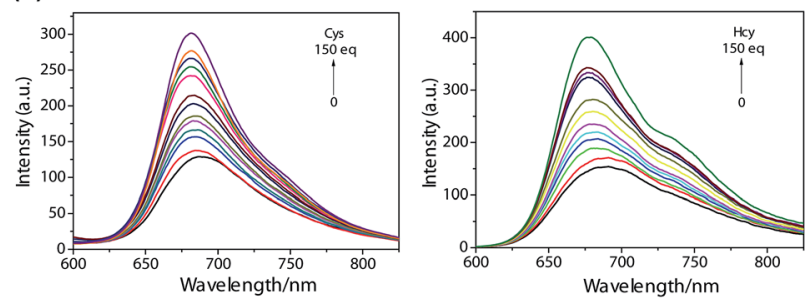

Fig. 1 Changes in absorption (a) and emission (b) spectra of NIR-Ir in DMSO-HEPES ( $4: 1 \mathrm{v} / \mathrm{v}$ ) upon addition of various amounts of Cys or Hcy. group that can quench the luminescence of $\operatorname{Ir}\left(\right.$ III) complex. ${ }^{26,27}$ After Cys or Hcy reacted with aldehyde groups in NIR-Ir, the emission intensity of $\operatorname{Ir}(\mathrm{III})$ complex enhanced. Moreover, the emission intensities of NIR-Ir $(5 \mu \mathrm{M})$ with different concentrations of Cys/Hcy show a linear correlation in the range of 10-90 equiv. of Cys/Hcy (Fig. S2, ESI $\dagger$ ), and a linear regression equation of $I=1.37 \mathrm{C}_{\mathrm{Cys}}+124.52$ (unit of $\mathrm{C}$ is $\mu \mathrm{M}, R=0.984$ ) for Cys and $I=1.94 \mathrm{C}_{\mathrm{Hcy}}+147.66$ (unit of $\mathrm{C}$ is $\mu \mathrm{M}, R=0.985$ ) for Hcy is obtained. The detection limit is calculated to be $13.7 \mu \mathrm{M}$ for Cys and $9.7 \mu \mathrm{M}$ for Hcy $(\mathrm{S} / \mathrm{N}=3)$, respectively. These facts indicate that the NIR-Ir could serve as an indicator of Cys/Hcy.

High selectivity is very important for an excellent chemosensor. Hence, studies of the selective response of NIR-Ir have been carried out with other elementary amino acids. The ratio of photoluminescent intensity at $684 \mathrm{~nm}\left(F / F_{0}\right)$ was selected to indicate the changes with or without addition of various amino acids. Amino acids such as Ala, Arg, Asp, Gln, Glu, Gly, GSH, His, Ile, Leu, Lys, Met, Phe, Pro, Ser, Thr, Trp, Tyr, and Val, did not produce detectable spectral changes (Fig. 2, black bars), indicating that the formation of thiazolidine and thiazinane was a key for the selective recognition of Cys and Hcy. Moreover, the competition experiments were performed to evaluate the specificity of NIR-Ir for Cys/Hcy over a complex mixture of amino acids. After the addition of 80 equiv. of Cys/Hcy to the above mixture, the phosphorescence intensity significantly increased (Fig. 2, blue and red bars). This competition experiment demonstrated that other elementary amino acids do not interfere with NIR-Ir phosphorescent detection of Cys/Hcy.

To seek further detailed information on the binding mechanism, the ${ }^{1} \mathrm{H}$ NMR interaction of NIR-Ir with Hcy/Cys was investigated. When excess Hcy or Cys was added to a solution of NIR-Ir in DMSO- $\mathrm{d}_{6}$, the aldehyde resonance (10.91 ppm) of NIRIr disappeared, which resulting from thiazinane (or thiazolidine) was formed by the interaction of aldehyde with Hcy (or

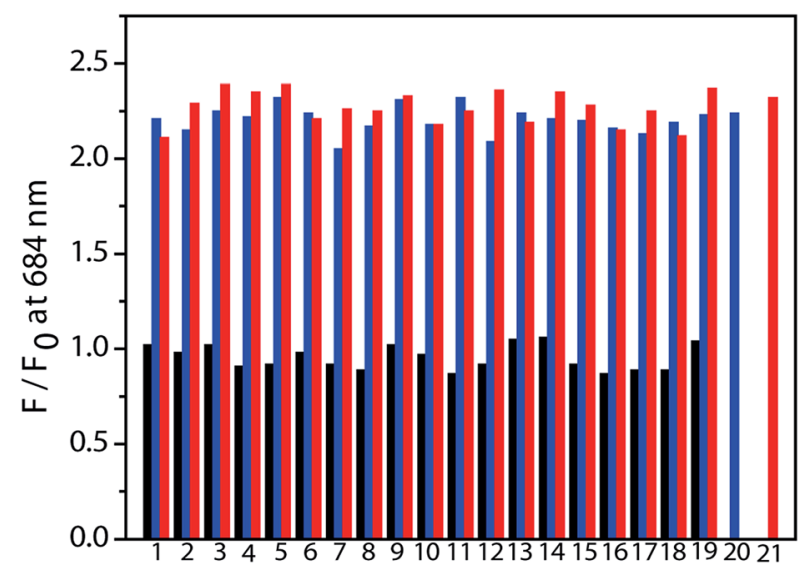

Fig. 2 Luminescent responses of NIR-Ir $(10 \mu \mathrm{M})$ in DMSO-HEPES $(4: 1 \mathrm{v} / \mathrm{v})$ to various amino acids $\left(80\right.$ equiv.) at $37^{\circ} \mathrm{C}$. Bars represent the ratio $F / F_{0}$ of luminescence intensity at $670 \mathrm{~nm}$. Black bars represent the addition of 80 -fold various amino acids to a $10 \mu \mathrm{M}$ solution of NIRIr. Blue and red bars represent the addition of Cys and Hcy (80 equiv.) to the above solution, respectively. (1) Gly; (2) Glu; (3) Gln; (4) GSH; (5) Met; (6) Arg; (7) Tyr; (8) Ala; (9) Lys; (10) Leu; (11) Pro; (12) Try; (13) Ser; (14) Thr; (15) Asp; (16) Asn; (17) Val; (18) Ile; (19) His; (20) Cys; (21) Hcy. 
Cys) (Fig. S3, ESI $\dagger$ ). ${ }^{27}$ Meanwhile, calculations based on density functional theory $(\text { DFT })^{\mathbf{4 5}}$ for NIR-Ir, NIR-Ir + Hcy and NIR-Ir + Cys were done to further understand the selectivity of NIR-Ir for detecting Hcy/Cys. Electron distributions of the frontier molecular orbitals (MOs) for these Ir(III) complexes are shown in Fig. S4 (ESI $\dagger$ ). Energies for these frontier MOs as well as their corresponding HOMO-LUMO gaps are listed in Table S2, $\uparrow$ TDDFT obtained vertical absorption and emission energies are collected in Table S3. $\uparrow$ TD-NTO analyses for hole-particle wave function pairs of natural transition orbital for the $S_{1}$ and $T_{1}$ states are displayed in Fig. S5 and S6 (ESI $\dagger$ ), respectively. For NIR-Ir, HOMO wave function is mainly distributed on the phenyl part of the cyclometalated ligands and the Ir center. The LUMO is localized on the $\mathrm{N}^{\wedge} \mathrm{N}$ ligand. Similar distributions of the frontier MOs are observed for NIR-Ir + Cys and NIR-Ir + Hcy. Calculated HOMO-LUMO energy gaps of NIR-Ir, NIR-Ir + Cys, and NIR-Ir + Hcy are 3.00, 3.19 and $3.04 \mathrm{eV}$, respectively. It means ligand modification has an effect on the frontier MOs. As a result, the vertical $S_{0} \rightarrow S_{1}$ absorption energies for these $\operatorname{Ir}(\mathrm{III})$ complexes are also influenced, because the $\mathrm{S}_{0} \rightarrow \mathrm{S}_{1}$ absorption is dominated by the HOMO to LUMO transition as obtained from further NTO analysis based on the optimized complexes in the ground state (Fig. S5, ESI $\dagger$ ). For the aim to get more information for emission, we also did NTO analyses for the holeparticle wave function pairs based on the optimized complexes in the triplet state. As shown in Fig. S6 (ESI $\dagger$ ), it proved that, for these $\operatorname{Ir}($ III) complexes, emission is mainly derived from the $\left[\pi \mathrm{C}^{\wedge} \mathrm{N}-\pi \mathrm{N}^{\wedge} \mathrm{N}^{*}\right]^{3} \mathrm{LLCT}$ and $\left[\mathrm{d} \pi(\mathrm{Ir})-\pi \mathrm{N}^{\wedge} \mathrm{N}^{*}\right]$ ${ }^{3}$ MLCT states.

A cellular level fluorescence probe should bear low cytotoxicity and the ability to enter the cell at working concentrations. Herein, the MTT assay was carried out (Fig. S7, ESI $\dagger$ ). After $24 \mathrm{~h}$ incubation with NIR-Ir, even at concentrations below $25 \mu \mathrm{M}$, which was five-fold the working concentration, the cell viabilities were above $80 \%$ signify that the probe with low cytotoxicity.

The ability of NIR-Ir to monitor Cys/Hcy within living cells was investigated by confocal fluorescence microscopy. The optical windows at the NIR channel 650-750 $\mathrm{nm}$ was chosen as signal output for detection. The reaction was allowed to proceed at $37^{\circ} \mathrm{C}$ for $30 \mathrm{~min}$ to obtain the adequate interaction between NIR-Ir and intracellular Cys/Hcy. As shown in Fig. 3a, MCF-7 cells incubated with $5 \mu \mathrm{M}$ NIR-Ir for $30 \mathrm{~min}$ at $37^{\circ} \mathrm{C}$ showed marked intracellular luminescence. By contrast, when MCF-7 cells were pretreated with $200 \mu \mathrm{M} \mathrm{N}$-ethylmaleimide (NEM, as a thio-reactive compound) at $37^{\circ} \mathrm{C}$ for $1 \mathrm{~h}$, and then incubated with complex NIR-Ir for $30 \mathrm{~min}$ at $37{ }^{\circ} \mathrm{C}$, MCF-7 cells showed very weak phosphorescence emission (Fig. $3 \mathrm{~b}$ ). Moreover, with the incubation time of NIR-Ir increased to $2 \mathrm{~h}$ after preincubation with $\mathrm{N}$-ethylmaleimide, the luminescence intensity increased (Fig. 3c), indicating the appearance of new Cys/Hcy in living MCF-7 cells and further reaction of NIR-Ir with the new Hcy/Cys, and NIR-Ir can detect the changes of Cys/Hcy level in the living cells. Furthermore, the photobleaching property when using NIR-Ir as an imaging probe was investigated at different excitation wavelengths. Under continuous irradiation at 543 or $405 \mathrm{~nm}$ with high power density for $10 \mathrm{~min}$, the photoluminescence intensity of NIR-Ir was maintained at $>90 \%$

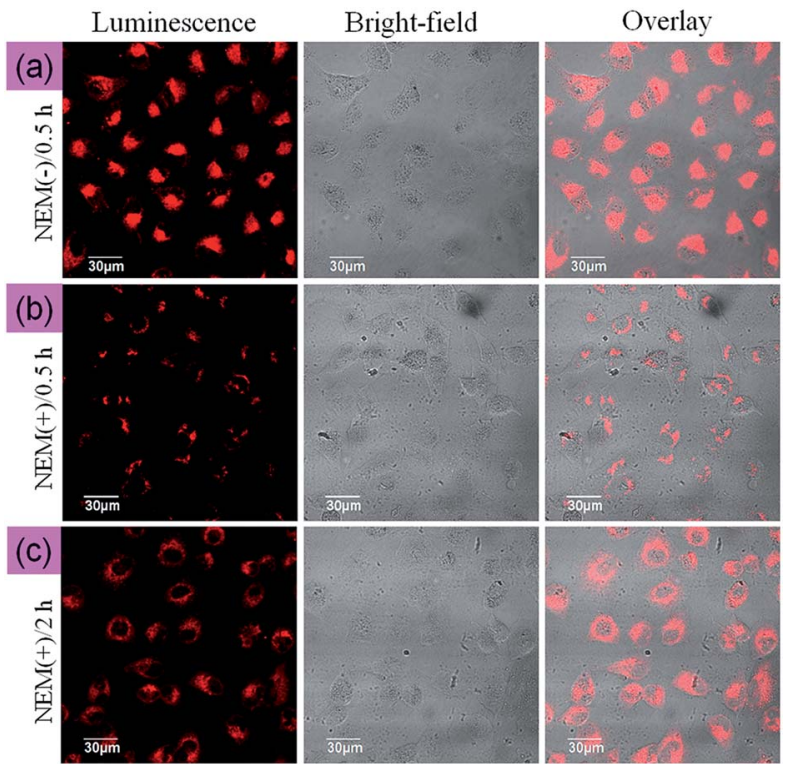

Fig. 3 Luminescence images of NIR-Ir-incubated MCF-7 cells, (a) MCF-7 cells incubated with $5 \mu \mathrm{M}$ NIR-Ir for $30 \mathrm{~min}$. (b) MCF-7 cells incubated with $200 \mu \mathrm{M} \mathrm{N}$-ethylmaleimide for $1 \mathrm{~h}$ and then further incubated with $5 \mu \mathrm{M}$ NIR-Ir for $30 \mathrm{~min}$. (c) MCF-7 cells incubated with $200 \mu \mathrm{M} \mathrm{N}$-ethylmaleimide for $1 \mathrm{~h}$ and then further incubated with $5 \mu \mathrm{M}$ NIR-Ir for $2 \mathrm{~h}$. Emission was collected by an NIR channel at $650-750 \mathrm{~nm}$, excitation at $\lambda_{\mathrm{ex}}=543 \mathrm{~nm}$.

and $\sim 75 \%$ of its initial value for $543 \mathrm{~nm}$ and $405 \mathrm{~nm}$, respectively (Fig. S8, ESI $\dagger$ ), indicating that excitation at long wavelength benefits the photo-stability of NIR-Ir in imaging.

Being encouraged by successful utilization of NIR-Ir in imaging Cys/Hcy in living cells, we evaluated the suitability of NIR-Ir for monitoring Cys in living mice. NIR-Ir $(20 \mu \mathrm{M}, 100 \mu \mathrm{L})$ and Cys $(200 \mu \mathrm{M}, 100 \mu \mathrm{L})$ were subcutaneously injected into a living mouse in order. As illustrated in Fig. 4a, after being

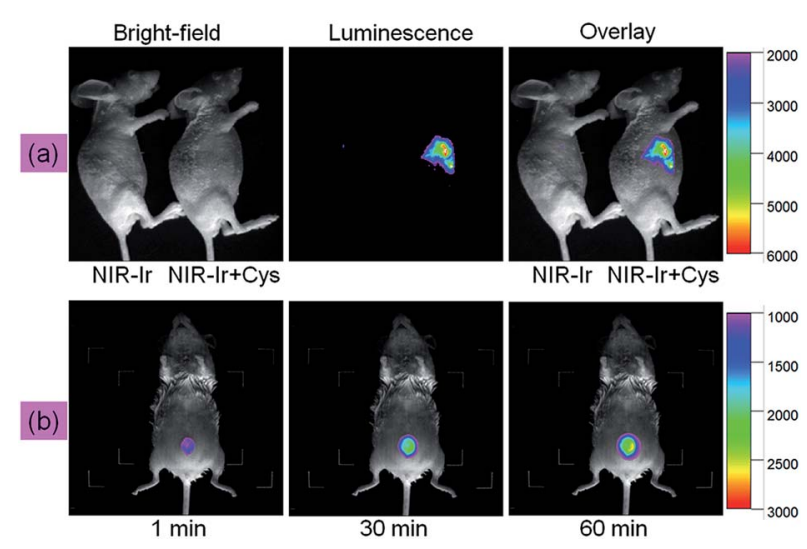

Fig. 4 (a) In vivo NIR images of NIR-Ir-pretreated living mice injected with $100 \mu \mathrm{L}$ normal saline (left mice) or $200 \mu \mathrm{M}$ Cys solution (right mice), the concentration of NIR-Ir is $20 \mu \mathrm{M}$. (b) Images of a shaved living mice that is given a subcutaneous injection of NIR-Ir $(20 \mu \mathrm{M}$, $100 \mu \mathrm{L})$ and Cys $(200 \mu \mathrm{M}, 100 \mu \mathrm{L})$. Images were taken after incubation for 1,30 , and $60 \mathrm{~min}$, respectively. The luminescence emission were collected at 650-750 $\mathrm{nm}$ as detection signals, upon irradiation at $532 \mathrm{~nm}$ laser. 
incubated for a certain time, luminescence intensity of right mouse increased over time, which can be contributed to the interaction of NIR-Ir with Cys. However, in the left control, no obvious fluorescence was observed under the same experimental condition. Furthermore, the emission intensity changes of NIR-Ir for Cys sensing with different time were investigated. A shaved living mice was preinjected subcutaneously with Cys $(200 \mu \mathrm{M}, 100 \mu \mathrm{L})$, and then treated with NIR-Ir $(20 \mu \mathrm{M}, 100 \mu \mathrm{L})$ via subcutaneous injection. As shown in Fig. 4b, the mice exhibited weakened emission signals within 1 min incubation, whereas the mice showed significantly stronger emission intensity with increasing the incubation time for $60 \mathrm{~min}$. The above results confirmed that the NIR probe NIR-Ir possessing the unique ability to monitor and image Cys in complicated living organisms.

In conclusion, a novel NIR-emitting iridium(III) complex NIRIr was designed and employed it as a switch-on chemosensor for detection and imaging of Cys/Hcy. NIR-Ir displays distinguishable advantages: compared with present iridium(III) complexes, it is a good candidate for detecting Cys/Hcy in vitro and in vivo (Table S4†). To the best of our knowledge, this is the first time that a NIR-emitting iridium(III) switch-on probe was used for imaging of Cys/Hcy in small animal (mice). Moreover, in order to eliminate auto-fluorescent from organism, NIR-Ir displays a large Stokes shift (>250 $\mathrm{nm}$ ) and a relatively long emission lifetime that allows it to be distinguished from background autofluorescent via the use of time-resolved photoluminescence technique (TRPT) or fluorescence lifetime imaging microscopy (FLIM). The present results offer valuable information and create a new platform for designing NIR-emitting probe based on phosphorescent metal complexes for in vivo imaging.

\section{Ethical statement}

"Animal procedures were in agreement with the guidelines of the Institutional Animal Care and Use Committee of Gannan Normal University and performed in accordance with the institutional guidelines for animal handling. All of the animal experiments were approved by the Science and Technology Committee of Jiangxi Province (China)”.

\section{Conflicts of interest}

There are no conflicts to declare.

\section{Acknowledgements}

This work was supported by Natural Science Foundation of China (21501031, 51478123, 21463003), the Key Technology R\&D Program of Jiangxi Province (20143ACG70019), and Natural Science Foundation of Jiangxi Province of China (20151BAB213001).

\section{Notes and references}

1 Z. A. Wood, E. Schröder, J. Robin Harris and L. B. Poole, Trends Biochem. Sci., 2003, 28, 32-40.
2 Y. Zhou and J. Yoon, Chem. Soc. Rev., 2012, 41, 52-67.

3 X. Chen, Y. Zhou, X. Peng and J. Yoon, Chem. Soc. Rev., 2010, 39, 2120-2135.

4 H. Refsum, P. M. Ueland, O. Nygård and S. E. Vollset, Annu. Rev. Med., 1998, 49, 31-62.

5 S. Seshadri, A. Beiser, J. Selhub, P. F. Jacques, I. H. Rosenberg, R. B. D'Agostino, P. W. F. Wilson and P. A. Wolf, N. Engl. J. Med., 2002, 346, 476-483.

6 Z. Guo, S. Nam, S. Park and J. Yoon, Chem. Sci., 2012, 3, 2760-2765.

7 Q. Gao, W. Zhang, B. Song, R. Zhang, W. Guo and J. Yuan, Anal. Chem., 2017, 89, 4517-4524.

8 O. Rusin, N. N. S. Luce, R. A. Agbaria, J. O. Escobedo, S. Jiang, I. M. Warner, F. B. Dawan, K. Lian and R. M. Strongin, J. Am. Chem. Soc., 2004, 126, 438-439.

9 (a) Q. Zhao, F. Li and C. Huang, Chem. Soc. Rev., 2010, 39, 3007-3030; (b) H. Shi, H. Sun, H. Yang, S. Liu, G. Jenkins, W. Feng, F. Li, Q. Zhao, B. Liu and W. Huang, Adv. Funct. Mater., 2013, 23, 3268-3276; (c) H. Shi, X. Ma, Q. Zhao, B. Liu, Q. Qu, Z. An, Y. Zhao and W. Huang, Adv. Funct. Mater., 2014, 24, 4823-4830; (d) S. J. Liu, Y. L. Zhang, H. Liang, Z. J. Chen, Z. Y. Liu and Q. Zhao, Opt. Express, 2016, 24, 15757; (e) S. J. Liu, N. Zhou, Z. J. Chen, H. J. Wei, Y. N. Zhu, S. Guo and Q. Zhao, Opt. Lett., 2017, 42, 13.

10 D.-L. Ma, V. P.-Y. Ma, D. S.-H. Chan, K.-H. Leung, H.-Z. He and C.-H. Leung, Coord. Chem. Rev., 2012, 256, 3087-3113.

11 Q. Zhao, C. Huang and F. Li, Chem. Soc. Rev., 2011, 40, 25082524.

12 D.-L. Ma, S. Lin, W. Wang, C. Yang and C.-H. Leung, Chem. Sci., 2017, 8, 878-889.

13 Y. Q. Wu, H. Jing, Z. S. Dong, Q. Zhao, H. Z. Wu and F. Y. Li, Inorg. Chem., 2011, 50, 7412-7420.

14 Y. Wu, M. Shi, L. Zhao, W. Feng, F. Li and C. Huang, Biomaterials, 2014, 35, 5830-5839.

15 L. Murphy, A. Congreve, L.-O. Palsson and J. A. G. Williams, Chem. Commun., 2010, 46, 8743-8745.

16 W. Wang, Z. Mao, M. Wang, L.-J. Liu, D. W. J. Kwong, C.-H. Leung and D.-L. Ma, Chem. Commun., 2016, 52, 3611-3614.

17 K. K.-S. Tso, K.-K. Leung, H.-W. Liu and K. K.-W. Lo, Chem. Commun., 2016, 52, 4557-4560.

18 J. Liu, C. Jin, B. Yuan, X. Liu, Y. Chen, L. Ji and H. Chao, Chem. Commun., 2017, 53, 2052-2055.

19 J. Yellol, S. A. Perez, G. Yellol, J. Zajac, A. Donaire, G. Vigueras, V. Novohradsky, C. Janiak, V. Brabec and J. Ruiz, Chem. Commun., 2016, 52, 14165-14168.

20 F. Zhang, X. Liang, W. Zhang, Y.-L. Wang, H. Wang, Y. H. Mohammed, B. Song, R. Zhang and J. Yuan, Biosens. Bioelectron., 2017, 87, 1005-1011.

21 W. Zhang, F. Zhang, Y.-L. Wang, B. Song, R. Zhang and J. Yuan, Inorg. Chem., 2017, 56, 1309-1318.

22 Y. Wu, G. Zeng, N. Lvyue, W. Wu, T. Jiang, R. Wu, W. Guo, X. Li and X. Fan, J. Mater. Chem. B, 2017, 5, 4973-4980.

23 T. S.-M. Tang, H.-W. Liu and K. K.-W. Lo, Chem. Commun., 2017, 53, 3299-3302.

24 H.-Y. Shiu, M.-K. Wong and C.-M. Che, Chem. Commun., 2011, 47, 4367-4369. 
25 Z. Mao, M. Wang, J. Liu, L.-J. Liu, S. M.-Y. Lee, C.-H. Leung and D.-L. Ma, Chem. Commun., 2016, 52, 4450-4453.

26 H. Chen, Q. Zhao, Y. Wu, F. Li, H. Yang, T. Yi and C. Huang, Inorg. Chem., 2007, 46, 11075-11081.

27 L. Xiong, Q. Zhao, H. Chen, Y. Wu, Z. Dong, Z. Zhou and F. Li, Inorg. Chem., 2010, 49, 6402-6408.

28 Y. Ma, S. Liu, H. Yang, Y. Wu, C. Yang, X. Liu, Q. Zhao, H. Wu, J. Liang, F. Li and W. Huang, J. Mater. Chem., 2011, 21, 18974-18982.

29 X. Cao, Y. Wu, K. Liu, X. Yu, B. Wu, H. Wu, Z. Gong and T. Yi, J. Mater. Chem., 2012, 22, 2650-2657.

30 X. Liu, N. Xi, S. Liu, Y. Ma, H. Yang, H. Li, J. He, Q. Zhao, F. Li and W. Huang, J. Mater. Chem., 2012, 22, 7894-7901.

31 S. Liu, W. Qiao, G. Cao, Y. Chen, Y. Ma, Y. Huang, X. Liu, W. Xu, Q. Zhao and W. Huang, Macromol. Rapid Commun., 2013, 34, 81-86.

32 Y. Ma, S. Liu, H. Yang, Y. Wu, H. Sun, J. Wang, Q. Zhao, F. Li and W. Huang, J. Mater. Chem. B, 2013, 1, 319-329.

33 Y. Tang, H.-R. Yang, H.-B. Sun, S.-J. Liu, J.-X. Wang, Q. Zhao, X.-M. Liu, W.-J. Xu, S.-B. Li and W. Huang, Chem.-Eur. J., 2013, 19, 1311-1319.

34 (a) W. Xu, X. Zhao, W. Lv, H. Yang, S. Liu, H. Liang, Z. Tu, H. Xu, W. Qiao, Q. Zhao and W. Huang, Adv. Healthcare Mater., 2014, 3, 658-669; (b) S. J. Liu, A. Q. Xu, Z. J. Chen,
Y. Ma, H. R. Yang, Z. J. Shi and Q. Zhao, Opt. Express, 2016, 24, 28247.

35 G. Li, Y. Chen, J. Wu, L. Ji and H. Chao, Chem. Commun., 2013, 49, 2040-2042.

36 N. Zhao, Y.-H. Wu, L.-X. Shi, Q.-P. Lin and Z.-N. Chen, Dalton Trans., 2010, 39, 8288-8295.

37 K. Huang, I. W. Bulik and A. A. Marti, Chem. Commun., 2012, 48, 11760-11762.

38 K. Chen and M. Schmittel, Analyst, 2013, 138, 6742-6745.

39 H. Gao, Z. Li, Y. Zhao, H. Qi and C. Zhang, Sens. Actuators, B, 2017, 245, 853-859.

40 H. Chen, B. Dong, Y. Tang and W. Lin, Acc. Chem. Res., 2017, 50, 1410-1422.

41 F. Kong, Z. Liang, D. Luan, X. Liu, K. Xu and B. Tang, Anal. Chem., 2016, 88, 6450-6456.

42 J.-Y. Xie, C.-Y. Li, Y.-F. Li, J. Fei, F. Xu, J. Ou-Yang and J. Liu, Anal. Chem., 2016, 88, 9746-9752.

43 J. H. Palmer, T. Brock-Nannestad, A. Mahammed, A. C. Durrell, D. VanderVelde, S. Virgil, Z. Gross and H. B. Gray, Angew. Chem., Int. Ed., 2011, 50, 9433-9436.

44 G. Zhang, H. Zhang, Y. Gao, R. Tao, L. Xin, J. Yi, F. Li, W. Liu and J. Qiao, Organometallics, 2014, 33, 61-68.

45 A. D. Becke, J. Chem. Phys., 1993, 98, 5648-5652. 